\title{
The situation of former adolescent self-injurers as young adults: a follow-up study

\author{
Rebecca C. Groschwitz ${ }^{1 \dagger}$, Paul L. Plener ${ }^{1 *}{ }^{*}$, Michael Kaess $^{2}$, Teresa Schumacher ${ }^{1}$, Ramona Stoehr ${ }^{3}$ \\ and Isabel Boege ${ }^{3}$
}

\begin{abstract}
Background: Nonsuicidal self-injury (NSSI) in adolescence has been described as comorbid condition in affective or anxiety disorders, as well as borderline personality disorder (BPD) and is a risk factor for later suicide attempts. Prevalence rates of NSSI decline steeply from adolescence to young adulthood. Yet, to the best of our knowledge, the longitudinal development of adolescent psychiatric patients with NSSI into their young adulthood has not been investigated. The aim of this study was to assess current NSSI and psychological impairment of young adults, who had been in treatment for NSSI in their adolescence.

Methods: Former patients of the departments of child and adolescent psychiatry and psychotherapy in Ulm and Ravensburg, Germany ( $N=52)$, who presented with NSSI in their adolescence, were recruited (average age: 21.5 years $(S D=2.6)$ ). Data was assessed using questionnaires and structured clinical interviews. Two groups of participants with prevailing NSSI and ceased NSSI were compared concerning their current psychological impairment, history of NSSI, suicide attempts, and BPD diagnosis.
\end{abstract}

Results: Around half of all participants had engaged in NSSI within the last year, and around half met diagnostic criteria for BPD. Although there was no significant association between current NSSI and BPD, an earlier age of onset of NSSI and a longer duration of NSSI during adolescence was significantly predictive of adult BPD. Two thirds of participants still met criteria of an axis 1 psychiatric disorder. Suicide attempts were reported by $53.8 \%$ of all participants. Participants with current NSSI were more likely to meet criteria for a current axis 1 disorder, had engaged in NSSI more often in their lifetime, and reported more suicide attempts.

Conclusions: Reduction of NSSI from adolescence to young adulthood was lower than described in previous community samples. This may be due to the initial high psychiatric impairment of this sample in adolescence. Early onset of NSSI seemed to be a risk factor for a longer duration of NSSI during adolescence but not for NSSI prevailing into adulthood. However, it was a risk factor for adult BPD. Furthermore, the occurrence of suicidal thoughts and behaviors and prevailing NSSI was highly associated.

Keywords: Non-suicidal self-injury, NSSI, Longitudinal, Follow-up

\section{Background}

Non-suicidal self-injury (NSSI) is defined as the deliberate destruction of body tissue without suicidal intent [1]. Whereas NSSI is mentioned as a symptom of borderline personality disorder (BPD), especially in adolescence, it most often exists comorbid to further psychopathology like depressive or anxiety symptoms [2, 3]. However, it

\footnotetext{
* Correspondence: paul.plener@uniklinik-ulm.de

${ }^{\dagger}$ Equal contributors

'Department of Child and Adolescent Psychiatry and Psychotherapy, University of Ulm, Steinhoevelstr. 5, 89075 Ulm, Germany

Full list of author information is available at the end of the article
}

can also occur without further psychopathology [4, 5]. Recent prevalence studies have pointed towards NSSI being of major concern among adolescents, with international lifetime prevalence rates of around $18 \%$ in the general population [6] and around $50 \%$ in psychiatric in-patient samples [7].

A decline of prevalence rates of deliberate self-harm from late adolescence to young adulthood was shown in one large longitudinal community-based study with 1943 participants followed up in seven waves from age 15 to age 29 [8]. Naturally, only a small percentage (8\%)

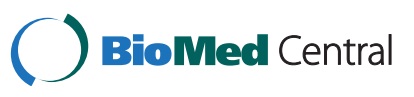


had engaged in self-harm at baseline and throughout the course of the study. Self-harm in young adulthood was significantly correlated with symptoms of anxiety and depression in adolescence.

In addition, a recent systematic review of longitudinal studies with sample sizes ranging from 49 to 936,449 participants provided evidence that NSSI seems to peak around the age of 16 years and decline throughout young adulthood [9]. Previous NSSI, depression, female gender, suicidality and psychological distress were among the most cited predictors for NSSI in the reviewed longitudinal studies. However, general assumptions about the longitudinal course of NSSI are not easily made, as studies included in this review were heterogenic regarding definitions of NSSI and its assessment, as well as timespans of follow-up periods and sample sizes.

Even though NSSI seems to decline in young adulthood, it has been shown to be a significant risk factor for suicide attempts in adolescents $[2,10,11]$ and adults [12]. However, to our knowledge, only two studies have investigated the predictive nature of adolescent NSSI and suicide attempts in young adulthood: Tuisku et al. [13] found NSSI to be a significant predictor for suicide attempts at the 1 year follow-up (with participants being around 17.5 years of age), and at the 8-year follow-up. The Pittsburgh Girls Study collected data on NSSI, suicidal ideation and suicide attempts between ages 13 and 21 , showing that a combination of suicidal ideation and NSSI increases the likelihood of a suicide attempt later on in life [14]. These findings seem to suggest that even though NSSI may decline over time, psychopathology tends to prevail.

As NSSI is mentioned as a symptom of BPD, and BPD usually also becomes apparent during adolescence [15], numerous studies assessed the link between the two. One of the first studies in a clinical sample of 89 adolescent psychiatric patients with recent NSSI reported a BPD prevalence of $51.7 \%$ [16]. Interestingly, the exact same percentage $(51.7 \%)$ of BPD was found in a more recent study in adolescent psychiatric patients $(N=198)$ who fulfilled criteria for NSSI disorder according to DSM-5 [2]. In a recent study of 36 adolescents and young adults from an outpatient psychotherapy clinic, BPD affective dysregulation symptoms were more associated with intrapersonal functions of NSSI. The same was true for interpersonal BPD symptoms [17].

Given the high clinical relevance of NSSI as comorbid factor in psychiatric disorders and its role as risk factor for suicidality, there seems to be a gap in the literature regarding the longitudinal course of NSSI. This especially holds true for adolescents with severe NSSI and comorbid psychiatric disorders, as most longitudinal research stems from community samples. To the best of our knowledge, no study so far has followed-up patients, who were treated in child and adolescent psychiatry for NSSI in their adolescence into young adulthood.

The objectives of this study were to assess psychosocial functioning, suicidality, current NSSI and BPD in young adults, who had presented with NSSI to departments for child and adolescent psychiatry and psychotherapy in their past.

\section{Methods}

The study was approved by the Institutional Review Board (IRB) of the University of Ulm and was carried out in accordance with the Declaration of Helsinki.

\section{Recruitment process}

This study was conducted with former patients of the departments of child and adolescent psychiatry and psychotherapy in Ulm and Ravensburg, Germany. In order to recruit participants, archived files of every patient who had ever been treated at those two services in the years between October 2001 (foundation date of the Department of child and adolescent psychiatry and psychotherapy in Ulm) and December 2013 (end of data collection), and was now between 18 and 30 years old, were scanned electronically for NSSI related search terms (German wording for NSSI, selfinjury, self-injurious behavior, self-mutilation, ICD-10 X78). Patients, in whose files at least one episode of NSSI had been recorded, were contacted. This procedure did not rely on self-reported identification of patients, but instead assured a clinically verified record of NSSI in medical reports, which is rather unique in NSSI research. We excluded participants who were currently still treated at one of the two services, and participants with insufficient knowledge of German language. However, participants with current ongoing psychotherapeutic/psychiatric treatment elsewhere were included in this study. Recruitment process took place in three separate waves over a time span of 2.5 years. Both, Ulm and Ravensburg, are mid-sized towns, surrounded by rural areas in the south of Germany. All participants, except for one participant with Turkish parents, were born in Germany.

In total, $N=545$ former patients were identified and contacted by mail. The letter informed the potential participants about the purpose of the study. If they were willing to participate, they were asked to give written consent, to fill in and to return (via a prepaid envelope) a number of questionnaires, which had been sent out with the letter. No incentive was offered for returning questionnaires. Participants were also asked to state whether they would be willing to participate in an interview, and in this case to provide their phone number or email address. If participants were interested, they were invited for a semistructured interview, which took place in Ulm or in Ravensburg. As an incentive for the interview, 25€ were offered. In case of no reply, addresses were updated using 
the official register of residents. However, due to legal regulations, only addresses of participants who still lived in the area of Ulm or Ravensburg could be retrieved.

\section{Materials}

Participants gave information about general demographics and stated if they were in current psychotherapeutic or psychiatric treatment. Clinical axis 1 diagnoses, obtained at initial presentation during adolescence, were retrieved from clinical files.

General psychological impairment was assessed by the Brief symptom inventory (BSI, [18]), a 53-Item self-report questionnaire, using a 5-point Likert scale. The Global Severity Index (GSI) indicates overall psychological distress. The clinical cut-off is at GSI $=0.59, T=61$. Present Axis 1 diagnosis were assessed using the Diagnostic Interview for Mental Disorders-short version (Mini-DIPS, [19]). The Mini-DIPS is a well-established structured interview which assesses axis 1 disorders according to DSM-IV. All comorbid diagnoses were recorded.

As affective disorders were the most common diagnoses in the clinical files of patients at an adolescent age, we decided to add another instrument for assessing current depressive symptoms (Beck Depression Inventory, second edition (BDI-II; [20]; German version: [21])). The BDI-II assess DSM-IV criteria of major depression in 21 items. Results are classified as minimal depression (0-13), mild depression (14-19), moderate depression (20-28) and severe depression (29-63) [21]. Personality disorders were diagnosed by using the well-established Structured Clinical Interview for DSM-IV Axis II Personality Disorders (SCID-II) [22]. As the association of NSSI and BPD was of most interest, only data from group comparisons of current NSSI and BPD, and no other personality disorders, are presented in this paper. The semi-structured Self-injurious thoughts and behaviors interview (SITBI; [23]) was used to gain detailed information about participants' NSSI and suicide attempts (present and lifetime). The interview consists of 169 items regarding frequency, functions and other characteristics of several self-injurious thoughts and behaviors. The German version (SITBI-G) showed good interrater reliability $(K=0.77)$ and construct validity $(K=0.89)$ scores for the assessment of NSSI in an adolescent sample of psychiatric inpatients [24]. To our knowledge, the SITBI has only been used in adolescent samples so far [23-25]. Since our study consisted of young adults only, comparable values of quality criteria can be expected. All interviews were conducted by trained clinical psychologists at master level or board certified child and adolescent child psychiatrists.

\section{Statistical analysis}

Statistical analyses were carried out using IBM SPSS Statistics 21. Differences between groups were examined using $\mathrm{Chi}^{2}$-tests and $t$-tests. In cases where data was not normally distributed, Mann-Whitney-U-tests were applied. Alpha levels were Bonferroni-corrected. For all significant results, effect sizes were calculated (Cohen's d for $t$-tests, $r$-values for $\mathrm{Chi}^{2}$ and Mann-Whitney-U-tests. $R$-values were then transformed into Cohen's $d$-values, to make data comparable).

\section{Results}

Of the $N=545$ former patients who were contacted, $N=11$ refused participation. $N=33$ patients had moved and their current addresses could not be retrieved. $N=430$ patients did not reply to the letter, although their addresses were up to date or had been updated. Of the $N=71$ participants who returned completed questionnaires, $N=52$ participated in an interview. Data of those 52 participants is presented in this paper. Socio-demographic data of participants who declined participation cannot be presented due to local IRB regulations.

\section{Socio-demographic characteristics of participants}

All participants had reported at least one episode of NSSI prior to or during their psychotherapeutic/psychiatric treatment. On average, participants had been discharged from the ward 4.8 years $(\mathrm{SD}=2.7)$ prior to the interview.

At the time of the interview, participants were on average $M=21.50(\mathrm{SD}=2.61 ; \min =18, \max =28)$ years old. The majority of participants were female $(94.2 \%, N=49)$. All participants had completed schooling. Secondary school was finished by $23.1 \%(N=12)$ after 9 years (German "Hauptschule"), $42.3 \%(N=22)$ after 10 years ("Realschule") and $34.6 \%(N=18)$ after 13 years of schooling ("Gymnasium"). At the time of evaluation, $50 \%(N=26)$ of participants were currently studying or in an apprenticeship, $27 \%(N=14)$ were employed and $19.2 \%(N=10)$ were unemployed. Most participants did not have children $(88.5 \%, N=46)$, three participants (5.8\%) had one child and one participant (1.9\%) had two children. Around half $(53.8 \%, N=28)$ of participants were single, $32.7 \%(N=17)$ were in a relationship and $9.6 \%(N=5)$ were married.

\section{Psychological impairment}

Around one third of participants $(36.5 \%, N=19)$ reported being in current psychotherapeutic treatment and $30.8 \%(N=16)$ reported taking psychotropic medication regularly. However, $53.8 \%(N=28)$ of all participants reported neither being in psychotherapeutic treatment nor to be taking psychotropic medication.

The average Global Severity Index of the BSI showed a clinically significant score across all participants (MGSI $=0.93, \mathrm{SD}=0.59 ; T=71 ; \min =0.09, \max =2.51$ ). However, $33 \%(N=17)$ of participants did not score in the clinically significant range $(\mathrm{GSI} \leq 0.59, T \leq 60)$. 
The average score of those participants who scored in a clinically significant range was at $\mathrm{M}-\mathrm{GSI}=1.17(\mathrm{SD}=0.53)$, $T=74$, which corresponds to the 99th percentile.

Concerning depressive symptoms, participants on average scored in a mild depressive range $(\mathrm{M}-\mathrm{BDI}=17.3$, $\mathrm{SD}=11.98 ;$ Median $=16.00 ; \min =1, \max =59)$. In detail, $36.5 \%(N=19)$ scored in a minimal range, $51.9 \%(N=27)$ scored in a mild depressive range, $13.5 \%(N=7)$ scored in a moderate range, and $15.4 \%(N=8)$ scored in a severely depressive range.

As adolescents, most participants had been diagnosed with major depression (see Table 1).

According to the MINI-DIPS, $28.8 \%(N=15)$ of participants did not meet a current axis 1 psychiatric disorder. In more detail, $30.8 \%(N=16)$ met criteria for one, $21.2 \%(N=11)$ for two, $13.5 \%(N=7)$ for three

Table 1 Axis 1 diagnoses of participants

\begin{tabular}{|c|c|c|c|c|c|c|}
\hline \multirow[t]{2}{*}{ Axis 1 diagnosis } & \multicolumn{2}{|c|}{$\begin{array}{l}\text { As } \\
\text { adolescents }\end{array}$} & \multicolumn{2}{|c|}{$\begin{array}{l}\text { As } \\
\text { young } \\
\text { adults }\end{array}$} & \multicolumn{2}{|c|}{ Retainment } \\
\hline & N & $\%$ & N & $\%$ & N & $\begin{array}{l}\text { Percent of } \\
\text { adolescent } \\
\text { diagnosis }\end{array}$ \\
\hline Major depression & 31 & 59.6 & 20 & 38.5 & 15 & 48.4 \\
\hline Anxiety disorder & 8 & 15.4 & 20 & 38.5 & 6 & 75.0 \\
\hline Adaptive disorder & 12 & 32.1 & - & - & - & - \\
\hline Posttraumatic stress disorder & 3 & 5.8 & 13 & 25 & 1 & 33.3 \\
\hline Eating disorder & 9 & 17.3 & 13 & 25 & 7 & 77.8 \\
\hline Hyperkinetic disorder & 3 & 5.8 & - & - & - & - \\
\hline Conduct disorder & 8 & 15.4 & - & - & - & - \\
\hline Alcohol/drug abuse & 4 & 7.7 & 8 & 15.4 & 2 & 50.0 \\
\hline Somatoform disorder & - & - & 12 & 32.1 & - & - \\
\hline $\begin{array}{l}\text { Obsessive compulsive } \\
\text { disorder }\end{array}$ & - & - & 4 & 7.7 & - & - \\
\hline \multicolumn{7}{|l|}{ Axis 2 diagnosis } \\
\hline Antisocial PD & - & - & 4 & 7.7 & - & - \\
\hline Avoidant PD & - & - & 9 & 17.3 & - & - \\
\hline Borderline PD & - & - & 24 & 46.2 & - & - \\
\hline Dependent PD & - & - & 3 & 5.8 & - & - \\
\hline Depressive PD & - & - & 10 & 19.2 & - & - \\
\hline Histrionic PD & - & - & 1 & 1.9 & - & - \\
\hline Narcissistic PD & - & - & 5 & 9.6 & - & - \\
\hline Negativistic PD & - & - & 8 & 15.4 & & \\
\hline Obsessive-Compulsive PD & - & - & 9 & 17.3 & - & - \\
\hline Paranoid PD & - & - & 7 & 13.5 & - & - \\
\hline Schizoid PD & - & - & 0 & 0 & - & - \\
\hline Schizotypical PD & - & - & 0 & 0 & - & - \\
\hline
\end{tabular}

Numbers exceeding $100 \%$ are due to comorbid diagnoses $P D$ personality disorder and $5.8 \%(N=3)$ for four current axis 1 psychiatric disorders. The most common diagnoses were current episodes of major depression and anxiety disorders (specific phobia were excluded from analyses) $(38.5 \%, N=20$ respectively; for details see Table 1). A correlation analysis of clinical diagnoses in adolescence and at present revealed the following significant (alpha $<0.003$ Bonferroni corrected) intercorrelations: anxiety disorders in adolescence were significantly correlated with somatoform disorders in young adulthood ( $r=0.40, p=0.003)$, as well as conduct disorders in adolescence with substance dependence in young adulthood $(r=0.56, p<0.001)$. Anxiety disorders $(75 \%, N=6)$ and eating disorders $(77.8 \%, N=7)$ had a high rate of retainment from adolescence to young adulthood.

Over half of all participants $(61.5 \%, N=32)$ were diagnosed with a personality disorder according to the SCID-II. The most common diagnosis was borderline personality disorder $(N=24,46.2 \%)$, followed by depressive personality disorder $(N=10,19.2 \%$, see Table 1$)$.

\section{Suicidal behaviors}

Out of all participants, $96.2 \%(N=50)$ reported having had suicidal thoughts in their lifetime, and $38.5 \%(N=20)$ reported having had suicidal thoughts within the last year. With regard to suicide attempts, $57.1 \%(N=28)$ of participants reported having attempted suicide at least once in their lifetime and $9.6 \%(N=5)$ reported having attempted suicide within the last year.

\section{Non-suicidal self-injury}

On average, participants stated a lifetime number of NSSI of $M=334.46$ episodes $(\mathrm{SD}=576.80 ; \min =1, \max =2000$ ). The median number of NSSI was $m=50.0$. The average age of onset was 13.87 years $(\mathrm{SD}=1.80 ; \min =9, \max =17)$. The most common method of NSSI was cutting $(N=48$, $92.3 \%)$, followed by scraping ( $N=27,51.9 \%)$, and manipulating a wound $(N=22,42.3 \%)$. While $23.1 \%(N=12)$ of participants had used only one method of NSSI, the majority had used multiple methods $(M=3.21, \mathrm{SD}=2.07$, $\min =1, \max =10$ ).

In total, $53.8 \%(N=28)$ of all participants had discontinued NSSI at least 1 year prior to the interview. Furthermore, $9.6 \%(N=5)$ of participants stated having thought about engaging in NSSI, but had been able to resist. Of those participants who had discontinued NSSI (at least within the last year), the average age of cessation was at $M=16.93$ years $(\mathrm{SD}=1.86, \min =14, \max =23)$.

Of the $46.2 \%(N=24)$ participants with prevailing NSSI, $41.7 \%(N=10)$ reported repetitive NSSI (on five or more occasions) within the last year. Out of those participants who had continued engaging in NSSI within the last year, the average number of episodes was $M=9.96(\mathrm{SD}=13.51)$ 
times (median number of episodes $=3.50, \quad \min =1$, $\max =50)$.

\section{Comparison of participants with and without prevailing NSSI}

Participants who reported at least one incident of NSSI within the last year $(N=24)$ were compared to those who had ceased from NSSI for at least 1 year prior to the interview $(N=28)$.

\section{Socio-demographic characteristics}

There was no significant difference in current age between participants who had discontinued NSSI and those with prevailing NSSI $(p=0.34, T=0.959)$. Furthermore, there were no statistically significant differences in participants who had continued or discontinued NSSI regarding their level of school education, employment status, and relationship status (see Table 2).

\section{Psychological impairment}

Participants with prevailing NSSI were significantly $(p<0.001$, Bonferroni corrected $)$ more likely to receive psychotherapeutic treatment $(62.5 \% \quad(N=15) \quad$ vs. $14.3 \%(N=4) ; d=1.2)$ and to be taking psychotropic medication $(58.3 \%(N=14)$ vs. $7.1 \%(N=2), d=1.3)$.

Concerning self-report questionnaires, participants with prevailing NSSI scored significantly higher in the BSI $(p=0.002, d=0.93)$, and the BDI-II $(p<0.001, d=1.1)$.

Participants with prevailing NSSI were significantly more likely to be diagnosed with any axis-1 disorder according to DSM-IV (95.8 \% $(N=23)$ vs. $50 \%(N=14) ; p$ $<0.001, d=1.2$ ). Also, they were diagnosed significantly more often with depressive disorders than participants who had stopped NSSI $(75 \%(N=18)$ vs. $7.1 \%(N=2)$, $p<0.001, d=1.9)$. There were no differences with regard to other psychiatric diagnoses (see Table 2).

\section{Suicidal behaviors and NSSI}

Regarding suicide attempts, participants with prevailing NSSI reported significantly more suicide attempts within their lifetime $(p=0.015, d=0.72)$, but not significantly more attempts within the last year.

In addition, participants with prevailing NSSI reported significantly more episodes of NSSI in their lifetime ( $p=0.010, d=0.7)$. Furthermore, they had engaged in NSSI for a significantly longer time $(p<0.001, d=1.5)$. There were no statistically significant differences between the two groups with regard to age of onset of NSSI or number of methods used (see Table 2).

\section{Relationship of characteristics in adolescence and young} adulthood and the course of NSSI

There were no statistically significant correlations of any psychiatric diagnoses in adolescence and the duration,
Table 2 Differences between young adults with and without prevailing NSSI

\begin{tabular}{|c|c|c|c|c|c|c|}
\hline & $\begin{array}{l}\text { Prevailing } \\
\text { NSSI }\end{array}$ & $\begin{array}{l}\text { Ceased } \\
\text { NSSI }\end{array}$ & & & & \\
\hline T-tests & $M(S D)$ & $M(S D)$ & $d f$ & $\mathrm{~T}$ & $p$ & $d$ \\
\hline Current age & $\begin{array}{l}21.1 \\
(2.5)\end{array}$ & $\begin{array}{l}21.8 \\
(2.7)\end{array}$ & 50 & 0.96 & 0.34 & - \\
\hline BSI-GSI & $\begin{array}{l}1.2 \\
(0.63)\end{array}$ & $\begin{array}{l}0.70 \\
(0.44)\end{array}$ & 49 & 4.02 & 0.002 & 1.1 \\
\hline BDI-II & $\begin{array}{l}23.6 \\
(12.4)\end{array}$ & $\begin{array}{l}11.7 \\
(8.4)\end{array}$ & 49 & 3.33 & $<0.001$ & 1.3 \\
\hline $\begin{array}{l}\text { Duration of NSSI } \\
\text { (years) }\end{array}$ & $\begin{array}{l}7.2 \\
(3.5)\end{array}$ & $\begin{array}{l}3.0 \\
(2.2)\end{array}$ & 50 & 5.26 & $<0.001$ & 1.5 \\
\hline Age of onset NSSI & $\begin{array}{l}13.8 \\
(1.9)\end{array}$ & $\begin{array}{l}14.0 \\
(1.7)\end{array}$ & 50 & 0.42 & 0.67 & - \\
\hline Mann-Whitney-U-Tests & rank-sum & rank-sum & & Z & $p$ & $d$ \\
\hline $\begin{array}{l}\text { Frequency NSSI } \\
\text { (lifetime) }\end{array}$ & 775.5 & 602.5 & & 2.57 & 0.010 & 0.77 \\
\hline $\begin{array}{l}\text { Frequency suicide } \\
\text { attempts (lifetime) }\end{array}$ & 761.0 & 617.0 & & 2.43 & 0.015 & 0.72 \\
\hline $\begin{array}{l}\text { Frequency suicide } \\
\text { attempts (last year) }\end{array}$ & 681.0 & 697.0 & & 1.62 & 0.106 & - \\
\hline$C h i^{2}$-tests & $N(\%)$ & $N(\%)$ & $d f$ & $\mathrm{Chi}^{2}$ & $p$ & $d$ \\
\hline Single & $\begin{array}{l}16 \\
(69.6)\end{array}$ & $\begin{array}{l}12 \\
(44.4)\end{array}$ & 1 & 3.18 & 0.093 & - \\
\hline Employed & $\begin{array}{l}19 \\
(82.6)\end{array}$ & $\begin{array}{l}21 \\
(78.8)\end{array}$ & 1 & 0.181 & 0.736 & - \\
\hline $\begin{array}{l}\text { Highest possible } \\
\text { school education } \\
\text { (Abitur) }\end{array}$ & $\begin{array}{l}8 \\
(33.3)\end{array}$ & $\begin{array}{l}10 \\
(35.7)\end{array}$ & 1 & 0.032 & 1.00 & - \\
\hline $\begin{array}{l}\text { Current } \\
\text { psychotherapeutic } \\
\text { treatment }\end{array}$ & $\begin{array}{l}15 \\
(62.5)\end{array}$ & $\begin{array}{l}4 \\
(14.3)\end{array}$ & 1 & 12.96 & $<0.001$ & 1.2 \\
\hline $\begin{array}{l}\text { Current psychotropic } \\
\text { medication }\end{array}$ & $\begin{array}{l}14 \\
(58.3)\end{array}$ & $\begin{array}{l}2 \\
(7.1)\end{array}$ & 1 & 15.90 & $<0.001$ & 1.3 \\
\hline $\begin{array}{l}\text { Current Axis } 1 \\
\text { disorder (Mini-DIPS) }\end{array}$ & $\begin{array}{l}23 \\
(95.8)\end{array}$ & $\begin{array}{l}14 \\
(50.0)\end{array}$ & 1 & 13.23 & $<0.001$ & 1.2 \\
\hline $\begin{array}{l}\text { Current Major } \\
\text { Depression }\end{array}$ & $\begin{array}{l}18 \\
(75.0)\end{array}$ & $\begin{array}{l}2 \\
(7.1)\end{array}$ & 1 & 25.14 & $<0.001$ & 1.9 \\
\hline $\begin{array}{l}\text { Boderline personality } \\
\text { disorder }\end{array}$ & $\begin{array}{l}12 \\
(50)\end{array}$ & $\begin{array}{l}12 \\
(50)\end{array}$ & 1 & 0.265 & 0.781 & - \\
\hline
\end{tabular}

Significance level of $p<0.05$ Bonferroni-corrected: significance $p<0.003$

frequency or cessation of NSSI up to the current age of participants.

However, a younger age of onset of NSSI was significantly correlated with a longer duration of NSSI (corrected for current age, $p<0.001, r=-0.73$, see Fig. 1). However, it was not correlated with a higher frequency of NSSI or current NSSI.

The duration of NSSI in years was significantly correlated with the frequency of NSSI (lifetime), the number of suicide plans (lifetime), current BDI-II scores, and the number of current axis 1 diagnoses (see Table 3). 


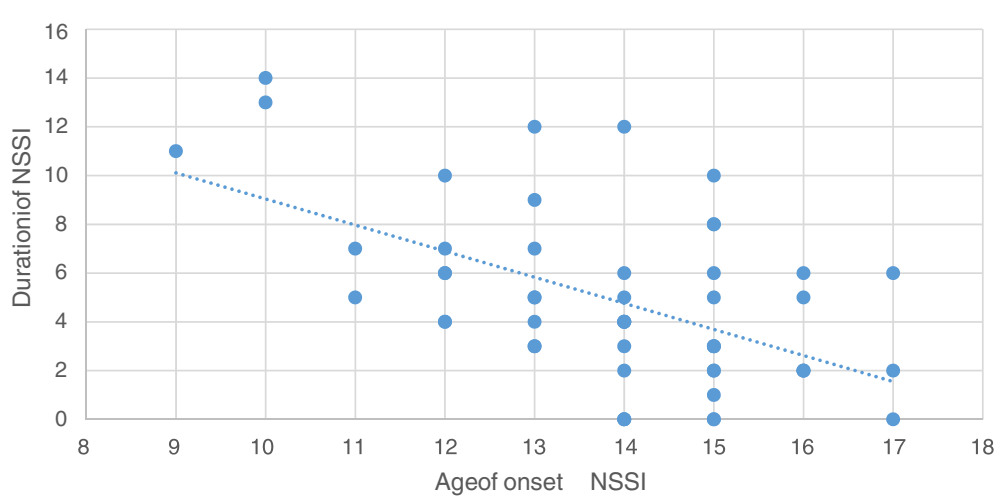

Fig. 1 Duration of NSSI in association with age of onset of NSSI

\section{Relationship of NSSI and borderline personality disorder (BPD)}

There was no observed relationship between current NSSI and current diagnosis of BPD (Chi $=0.265, p=0.78$, see Table 2). Of those participants currently diagnosed with BPD $(N=24,46.2 \%)$, exactly $50 \%(N=12)$ reported NSSI within the last year, while the other $50 \%$ did not (see Table 2). Of those participants without BPD $(N=28$, $53.8 \%), 42.9 \%(N=12)$ reported current NSSI, while $57.1 \%(N=16)$ did not.

However, there was a significant association $(Z=2.699$, $p=0.007, d=0.82$ ) between the age of onset of NSSI and a current diagnosis of BPD. Participants with BPD had started NSSI significantly earlier in life $(M=13.12$ years, $\mathrm{SD}=2.01)$ than adolescents without BPD $(M=14.50$ years,

Table 3 Correlation of duration of NSSI with other characteristics

\begin{tabular}{llll}
\hline & \multicolumn{3}{l}{$\begin{array}{l}\text { Duration of NSSI } \\
\text { (years) }\end{array}$} \\
\cline { 2 - 4 } Pearson correlation (2-tailed) & $N$ & $r$ & $p$ \\
\hline Current age & 52 & 0.34 & 0.013 \\
Age of onset NSSI & 52 & -0.55 & $<0.001$ \\
Age of cessation NSSI & 52 & 0.86 & $<0.001$ \\
Frequency NSSI (lifetime) & 52 & 0.46 & 0.001 \\
Frequency NSSI (last year) & 52 & 0.37 & 0.007 \\
Frequency suicide plans (lifetime) & 52 & 0.41 & 0.002 \\
Frequency suicide attempts (lifetime) & 52 & 0.39 & 0.004 \\
BSI-GSI & 51 & 0.31 & 0.026 \\
BDI-II & 51 & 0.42 & 0.002 \\
Number axis 1 diagnoses (Mini-DIPS) & 52 & 0.40 & 0.003 \\
Social support by at least one adult during & 52 & -0.30 & 0.033 \\
childhood/adolescence & & & \\
Current psychotherapeutic treatment & 52 & 0.23 & 0.108 \\
Current psychotropic medication & 52 & 0.34 & 0.014 \\
Boderline Personality Disorder & 52 & 0.43 & 0.001 \\
\hline Pearson corryyyyy
\end{tabular}

Pearson correlation, $p<0.05$ Bonferroni-corrected: significance $p<0.003$
$\mathrm{SD}=1.35)$. A similar association was found between $\mathrm{BPD}$ and duration of NSSI $(Z=2.93, p=0.003, d=0.94)$, Participants with BPD had engaged in NSSI for a significantly longer time $(M=6.54$ years, $\mathrm{SD}=3.71)$ than participants without $\mathrm{BPD}(M=3.50$ years, $\mathrm{SD}=2.76)$ as was also shown by a positive correlation of BPD and duration of NSSI $(r=0.43, p=0.001$, see Table 3$)$.

\section{Discussion}

In this naturalistic follow-up study, former adolescent psychiatric patients, who had engaged in self-injury, were examined with regards to their current psychosocial functioning and psychopathology as young adults. Results of this study showed a weaker decline of NSSI from adolescence to young adulthood as has been shown in studies of the general population, with around $50 \%$ still engaging in NSSI. Participants in this study generally still reported high psychiatric impairment. Prevailing NSSI was associated with greater current psychiatric impairment and a higher risk for suicide ideation. Although an early onset of NSSI did not seem to be a predictor for prevailing NSSI in young adulthood, it was significantly correlated to a longer duration of NSSI during adolescence and BPD in young adulthood.

\section{Psychiatric impairment}

At assessment, only around one third of all participants were free of general psychopathology (as defined by not meeting clinically significant scores in self-assessment tools) and did not meet current axis 1 diagnoses in a clinical structured interview. The rate of current axis 1 diagnoses of around $70 \%$ in this sample is by far higher than the $25-30 \%$ estimated 12-month prevalence in the general population [26]. This may be explained by the fact that this is a very selected population as the initial sample consisted of highly impaired former adolescent psychiatric in-patients. It is therefore on the other hand more than noteworthy that $30 \%$ did not meet any diagnosis anymore. At the same time, $53.8 \%$ of participants 
did not take psychotropic medication and were not treated by a psychotherapist anymore. This could either point to the fact that around $20 \%$ of participants are coping without treatment despite an axis 1 disorder, or to some percentage of participants not receiving professional help despite being in need for it. Non-treatment could furthermore point to the fact that there is a gap between child and adolescent and adult systems of psychiatric care in Germany, often leaving patients lost during their transition from childhood to adolescence [27].

\section{Socio-demographic characteristics}

The level of education was slightly lower than in the general population aged $20-25$ (23.1 vs. $17.2 \%$ "Hauptschule", 42.3 vs. $30.3 \%$ "Realschule", and 34.6 vs. $46.2 \%$ Gymnasium) [28]. Furthermore, the unemployment rate of $20 \%$ in this sample was also higher than the rate of around $6.7 \%$ in the general population [29]. Conclusions of whether adolescent psychiatric impairment is associated with educational underachievement and higher unemployment rates cannot be drawn from this small sample, especially when taking the participation rate of $10 \%$ into account. Rather, results may have been biased by favoring participation of unemployed adults who had more time at hand or were more drawn by the monetary incentive. However, results are in line with current research. Fergusson and Woodward [30] have shown a significantly increased risk for later educational underachievement and unemployment in adolescents with a major depression in a longitudinal study with 1265 participants aged $14-16$ at baseline.

\section{Course of NSSI}

Around half of all participants (46.2 \%) still reported at least one episode of NSSI within the last year. Surprisingly, there was no age difference between participants who had ceased from engaging in NSSI and those who still self-injured. It has to be taken in account though, that with an average age of 21.5 years, this sample was still quite young. According to the literature [8,9], it would have been expected for a larger number of participants to have ceased from NSSI by the age of 21. Again though, the low participation rate may have favored participants with prevailing NSSI to take part in the study. Furthermore, the population was not community based but consisted of individuals who had self-injured in a degree demanding inpatient care in their adolescence. It would be of great interest to conduct a long-term follow-up with these participants to investigate their long-term development.

Of those participants who had stopped engaging in NSSI, the average age of cessation was at 16.9 years. This is in accordance with Moran et al. [8] who found a significant drop of rates of self-harm between 15.9 and 17.4 years of age.

\section{NSSI and suicidality}

In accordance with previous findings (i.e. [2, 12]) NSSI and suicidal thoughts and behaviors were strongly interlinked. Almost all (96.2 \%) participants (who had all engaged in NSSI in their lives) reported suicidal thoughts in their lifetime and around half of them had tried to commit suicide at least once. Furthermore, in accordance with Tuisku et al. [13], those participants who still engaged in self-injury, reported more suicide attempts in their lives.

\section{Associative features of prevailing NSSI}

In longitudinal studies, the most commonly described predictor for future NSSI has been previous NSSI [9]. Our findings add on to this outcome, since participants who still engaged in NSSI reported a longer duration and a higher frequency of NSSI throughout their lifetime. Interestingly, adolescents with an earlier onset of NSSI also reported a longer duration of NSSI in years during adolescence but not into their young adulthood. Therefore, early onset of NSSI might be a risk factor for a longer duration of NSSI throughout adolescence.

In a number of studies, depressive symptoms have been identified as predictor for NSSI [9]. Results of our study support a link between NSSI and depression, with participants still engaging in NSSI being significantly more likely to meet criteria of a current major depression and scored significantly higher in the BDI-II.

Participants with prevailing NSSI also scored significantly higher in the BSI, were more likely to be diagnosed with an axis 1 disorder, and were more likely to receive psychotherapeutic treatment or to be taking psychotropic medication. Prevailing NSSI from adolescence to young adulthood might therefore be a risk factor for overall psychological impairment. However, NSSI might also serve as a coping mechanism to deal with ongoing psychiatric symptoms.

\section{NSSI and BPD}

We found a high prevalence rate of BPD in our sample of former adolescent psychiatric patients with NSSI. The rate of BPD diagnosis was $46.2 \%$, and slightly lower than the $51.7 \%$ in previously investigated adolescent psychiatric samples with NSSI $[2,16]$. Interestingly, in young adulthood, current NSSI was not associated with BPD diagnosis in our sample. However, adolescents with early onset of NSSI and long duration of NSSI during adolescence presented were at elevated risk to present with an adult diagnosis of BPD. The results may confirm the previously highlighted importance of developmental trajectories of NSSI and other self-harm behaviors as potential early markers of risk that may be used for early detection and intervention of BPD [15]. They may also well fit to a previous study indicating that first-presentation of BPD 
symptoms at young age could indicate a more severe prognosis of BPD [31].

\section{Limitations}

Limitations of this study were a predominantly female sample, which did not allow to draw conclusions on possible gender differences. The overall small sample size disallowed further analyses (i.e. regression analyses). Furthermore, the low participation rate of around $10 \%$ has to be taken into consideration, when interpreting results of this study. Therefore, results may have been biased by self-selection of participants (i.e. being interested in participating due to ongoing NSSI or declining participation due to high current psychiatric impairment). Further practical reasons (i.e. full-time employment, moving to other parts of Germany) may have led to declining participation. A number of possible participants could not be contacted, as due to legal privacy protection, current addresses could only be retrieved from participants who still lived in the state the study was conducted in. The majority of possible participants probably had moved away from their parents' house after finishing school. Therefore, a low participation rate of former adolescent psychiatric inpatients is a commonly reported problem in follow-up studies [12]. In addition, data on lifetime NSSI and lifetime suicidal behavior was assessed retrospectively which may have distorted the results. However, all participants had been former psychiatric inpatients, and former clinical diagnosis and medical evidence of NSSI were available from reliable electronic archive systems, avoiding adolescent NSSI status to rely on self-report only.

\section{Conclusions}

Overall, results of this study suggest a slight decrease of NSSI from adolescence to young adulthood in people with high psychiatric impairment and NSSI in adolescence. Early onset of NSSI seems to be a risk factor for a longer duration of NSSI in adolescence and an adult diagnosis of BPD, but not for prevailing NSSI in young adulthood. Furthermore, prevailing NSSI in young adulthood is associated with a greater risk for depression, suicide ideation, and other psychological impairment.

This exploratory study is, to our knowledge, the first study to follow up highly impaired adolescent psychiatric patients with NSSI into their young adulthood. Especially the relationship of NSSI in adolescence and its association with BPD later on in life is under researched. Although results are limited due to the small sample size and low participation rate, first conclusions can be drawn towards an association of early onset of NSSI and higher risk BPD in young adulthood. Larger longitudinal studies with clinical as well as healthy control groups would be needed to further investigate this question in more detail.

\section{Competing interests}

PP declares no competing interests. He received research grants from the BMBF (German Ministries for Research and Education), the BfArM (German Federal Institute for Drugs and Medical devices), the Baden Wuerttemberg foundation and the foundation for outpatient child and adolescent psychiatry. He was a PI in a study for Lundbeck. He received travel grants from the DFG, DAAD and IACAPAP. He isn't stockholder or share-holder in the pharmaceutical industry.

The other authors declare that they have no competing interests.

\section{Authors' contributions}

RG participated in acquiring data, performed the statistical analyses and drafted the manuscript. PP conceived of the study, participated in its design and coordination, participated in acquiring data and revised the manuscript critically for important intellectual content. MK participated in designing the study and revised the manuscript critically. TS participated in acquiring data. RS participated in the coordination of the study. IB participated in the coordination and acquisition of data of this study and revised the manuscript critically for important intellectual content. All authors read and approved the final manuscript.

\section{Authors' information}

Rebecca C. Groschwitz and Paul L. Plener Shared first authorship.

\section{Acknowledgements}

Funding for this project was provided by internal funds of the Dept. of Child and Adolescent Psychiatry and Psychotherapy at the University of Ulm.

\section{Author details}

${ }^{1}$ Department of Child and Adolescent Psychiatry and Psychotherapy, University of Ulm, Steinhoevelstr. 5, 89075 Ulm, Germany. ${ }^{2}$ Department of Child and Adolescent Psychiatry, Centre for Psychosocial Medicine, University Hospital Heidelberg, Blumenstr. 8, 69115 Heidelberg, Germany. ${ }^{3}$ Department of Child and Adolescent Psychiatry Weissenau, Centre for Psychiatry in South-Württemberg, Weingartshofer Straße 2, 88214 Ravensburg-Weissenau, Germany.

Received: 13 February 2015 Accepted: 14 July 2015

Published online: 18 July 2015

\section{References}

1. American Psychiatric Association. Diagnostic and statistical manual of mental disorders. 5th ed. Washington DC: American Psychiatric Association; 2013.

2. Glenn CR, Klonsky ED. Nonsuicidal self-injury disorder: an empirical investigation in adolescent psychiatric patients. J Clin Child Adolesc Psychol. 2013;42(4):496-507.

3. Jacobson CM, Gould M. The epidemiology and phenomenology of non-suicidal self-injurious behavior among adolescents: a critical review of the literature. Arch Suicide Res. 2007;11(2):129-47.

4. Klonsky ED. Non-suicidal self-injury in United States adults: prevalence, sociodemographics, topography and functions. Psychol Med. 2011;41(9):1981-6.

5. Stanford S, Jones MP. Psychological subtyping finds pathological, impulsive, and 'normal' groups among adolescents who self-harm. J Child Psychol Psychiatry. 2009;50(7):807-15.

6. Muehlenkamp JJ, Claes L, Havertape L, Plener PL. International prevalence of adolescent non-suicidal self-injury and deliberate self-harm. Child Adolesc Psychiatry Ment Health. 2012;6:10

7. Kaess M, Parzer P, Mattern M, Plener PL, Bifulco A, Resch F, et al. Adverse childhood experiences and their impact on frequency, severity, and the individual function of nonsuicidal self-injury in youth. Psychiatry Res. 2013;206(2-3):265-72.

8. Moran P, Coffey C, Romaniuk H, Olsson C, Borschmann R, Carlin JB, et al. The natural history of self-harm from adolescence to young adulthood: a population-based cohort study. Lancet. 2012;379(9812):236-43.

9. Plener P, Schumacher T, Munz L, Groschwitz R. The longitudinal course of non-suicidal self-injury and deliberate self-harm: a systematic review of the literature. Borderline Personality Disorder and Emotion Dysregulation. 2015;2(1):2. 
10. Guan K, Fox KR, Prinstein MJ. Nonsuicidal self-injury as a time-invariant predictor of adolescent suicide ideation and attempts in a diverse community sample. J Consult Clin Psychol. 2012;80(5):842-9.

11. Wilkinson P, Kelvin R, Roberts C, Dubicka B, Goodyer I. Clinical and psychosocial predictors of suicide attempts and nonsuicidal self-injury in the Adolescent Depression Antidepressants and Psychotherapy Trial (ADAPT). Am J Psychiatry. 2011;168(5):495-501.

12. Sinclair JM, Hawton K, Gray A. Six year follow-up of a clinical sample of self-harm patients. J Affect Disord. 2010;121(3):247-52.

13. Tuisku V, Kiviruusu O, Pelkonen M, Karlsson L, Strandholm T, Marttunen M. Depressed adolescents as young adults-predictors of suicide attempt and non-suicidal self-injury during an 8-year follow-up. J Affect Disord. 2014:152-154:313-9.

14. Scott LN, Pilkonis PA, Hipwell AE, Keenan K, Stepp SD. Non-suicidal self-injury and suicidal ideation as predictors of suicide attempts in adolescent girls: A multi-wave prospective study. Compr Psychiatry. 2015;58:1-10

15. Kaess M, Brunner R, Chanen A. Borderline personality disorder in adolescence. Pediatrics. 2014;134:782-93.

16. Nock MK, Joiner TE, Gordon KH, Lloyd-Richardson E, Prinstein MJ. Non-suicidal self-injury among adolescents: diagnostic correlates and relation to suicide attempts. Psychiatry Res. 2006;144:65-72.

17. Sadeh N, Londahl-Schaller EA, Piatigorsky A, Fordwood S, Stuart BK, McNiel DE, et al. Functions of non-suicidal self-injury in adolescents and young adults with Borderline Personality Disorder symptoms. Psychiatry Res. 2014;216:217-22.

18. Derogitas LR. BSI brief symptom inventory: administration, scoring, and procedure manual. 4th ed. Minneapolis, MN: National Computer Systems; 1993.

19. Margraf J. Mini-DIPS: Diagnostisches Kurz-Interview bei psychischen Störungen. Berlin: Springer; 1994.

20. Beck AT, Steer RA, Brown GK. Manual for the beck depression inventory-II. San Antonio, Texas: Psychological Corporation; 1996.

21. Hautzinger M, Keller F, Kühner C. BDI-II Beck Depressions-Inventar. Frankfurt am Main: Harcourt Test Services; 2006.

22. First MB, Gibbon M, Spitzer RL, Williams JBW, Benjamin LS. Structured clinical interview for DSM-IV axis II personality disorders (SCID-II). Washington DC: American Psychiatric Press, Inc; 1997.

23. Nock MK, Holmberg EB, Photos VI, Michel BD. Self-injurious thoughts and behaviors interview: development, reliability, and validity in an adolescent sample. Psychol Assess. 2007;19(3):309-17.

24. Fischer G, Ameis N, Parzer P, Plener PL, Groschwitz R, Vonderlin E, et al. The German version of self-injurious thoughts and behaviors interview (SITBI-G) a tool to assess non-suicidal self-injury and sucidal behavior disorder. BMC Psychiatry. 2014;14:265

25. Garcia-Nieto R, Carballo JJ, Hernando MD, de Léon-Martinez V, Baca-Garcia E. Clinical correlates of non-suicidal self-injury (NSSI) in an outpatient sample of adolescents. Arch Suicide Res. 2015;19(2):218-30.

26. Jacobi F, Kessler-Scheil S. Epidemiologie psychischer Störungen. Häufigkeit und Krankheitslast in Deutschland. Psychotherapeut. 2013;58:191-206.

27. Plener PL, Groschwitz RC, Fegert JM, Freyberger HJ: Die stationäre psychiatrische Versorgung Adoleszenter in Deutschland. Zeitschrift für Psychiatrie, Psychologie und Psychotherapie. 2015;63:181-87.

28. Statistisches Bundesamt. Bevölkerung 2013 nach Bildungsabschluss und Altersgruppen in Deutschland. 2015.

29. Statistisches Bundesamt. Registrierte Arbeitslose, Arbeitslosenquote1 nach Gebietsstand. 2015.

30. Fergusson DM, Woodward LJ. Mental health, educational, and social role outcomes of adolescents with depression. Arch Gen Psychiatry. 2002;59:225-31.

31. Chanen AM, Jovev M, Jackson HJ. Adaptive functioning and psychiatric symptoms in adolescents with borderline personality disorder. J Clin Psychiatry. 2007;68:297-306.

\section{Submit your next manuscript to BioMed Central and take full advantage of:}

- Convenient online submission

- Thorough peer review

- No space constraints or color figure charges

- Immediate publication on acceptance

- Inclusion in PubMed, CAS, Scopus and Google Scholar

- Research which is freely available for redistribution 